\title{
RELACIONES PÚBLICAS: PATROCINIO Y MECENAZGO
}

\section{PUBLIC RELATIONS: SPONSORSHIP AND PATRONAGE}

Luis Felipe Solano Santos: Facultad de Ciencias de la Información de la Universidad Complutense de Madrid. (España)

1fsolano@yahoo.es

\section{CURRÍCULUM VITAE}

Doctor en Ciencias de la Información. Licenciado en Periodismo por la Facultad de Ciencias de la Información de la Universidad Complutense de Madrid (España). Es miembro de un Grupo de Investigación Validado Complutense y director de varios proyectos de investigación. Ponente en varios Congresos nacionales e internacionales. Ha publicado tres libros y numerosos artículos en libros y revistas científicas.

\section{RESUMEN}

El presente artículo aborda cómo las acciones de Patrocinio y Mecenazgo, basadas en la aplicación de técnicas de Relaciones Públicas, forman parte de ese conjunto de obligaciones inherentes a las organizaciones empresariales tanto públicas como privadas, más concretamente, entes colectivos formales. Es decir, se trata de manifestaciones de reconocimiento de responsabilidad social corporativa que indudablemente generan una reputación social óptima, además de una gran rentabilidad económica para las mismas. 


\title{
PALABRAS CLAVE
}

Relaciones Públicas - Patrocinio - Mecenazgo

\begin{abstract}
The present article approaches how the technologies of Sponsorship and Patronage, instruments of Public Relations, form a part of this set of obligations inherent in the managerial organizations both public and deprived, more concretely, collective formal entities. That is to say, it is a question of expressions of recognition of corporate social responsibility that undoubtedly they generate a social ideal reputation, besides great economic profitability.
\end{abstract}

\section{KEY WORDS}

Public relations - Sponsorship - Patronage

\section{ÍNDICE}

1. Introducción

2. Patrocinio y Mecenazgo: Técnicas de Relaciones Públicas

3. Patrocinio y Mecenazgo, instrumentos de Responsabilidad Social Corporativa

4. Conclusiones

5. Bibliografía

TEXTO 


\section{Introducción}

¿Qué son el Patrocinio y el Mecenazgo? ¿Qué es el Patrocinio? ¿Qué es el Mecenazgo? ¿Son lo mismo? ¿No? Y si no son lo mismo, ¿Por qué razón van siempre de la mano? Éstas y otras cuestiones son las que pretenden aclararse a lo largo de las próximas páginas, además de subrayar y abordar su máxima relevancia en el ámbito de las Relaciones Públicas y, por supuesto, en el de la denominada Responsabilidad Social Corporativa. ¿Acaso no son el Patrocinio y Mecenazgo técnicas de Relaciones Públicas? Sin duda alguna, pues designan procesos de comunicación específicos que las organizaciones desarrollan y ponen en práctica al establecer unas "Relaciones Públicas" adecuadas, provechosas y beneficiosas. ¿Acaso no son expresión de reconocimiento de Responsabilidad Social? No nos cabe la menor duda, pues no en vano el Mecenazgo es el primer precedente histórico en este sentido. Ahí están los denodados esfuerzos de tantas y tantas figuras a lo largo de la historia encaminados a impulsar las artes favoreciendo a artistas, verbigracia, Cayo Cilnio Mecenas a Horacio y Virgilio; el emperador Trajano a Plinio el Joven; Alfonso X el sabio a los integrantes de la Escuela de Traductores de Toledo; Lorenzo el Magnífico a Miguel Ángel; el Duque de Ferrara a Tasso... Actitudes o acciones aparentemente filantrópicas o consideradas como tales en un principio, pero que el transcurso del tiempo ha revelado que su origen o naturaleza se halle más en el requerimiento social que en el amor que unos profesan a sus semejantes. Por lo tanto, el Patrocinio y Mecenazgo son, en primer lugar, técnicas de Relaciones Públicas; en segundo lugar, manifestaciones de Responsabilidad Social; y por último, instrumentos de creación de imagen que reportan una gran rentabilidad.

\section{Patrocinio y Mecenazgo: Técnicas de Relaciones Públicas}


¿Qué es el Patrocinio? El Patrocinio es la ayuda económica, o de otro tipo, generalmente con fines publicitarios, que se da a una persona para que pueda realizar una actividad; mientras que el Mecenazgo es la protección dada a las artes y la cultura. Y más concretamente, el Patrocinio es una técnica de comunicación estructurada, mediante la cual una empresa -patrocinador- ofrece unos recursos en forma monetaria o en especie a una organización, evento o causa -patrocinado- con la finalidad de obtener un beneficio directo al asocial su imagen, corporativa o de marca, sus productos o servicios, a la actividad o entidad patrocinada; mientras que el concepto originario del Mecenazgo responde a la vocación de la empresa de revertir a la sociedad parte de los beneficios que obtiene de ésta, en forma de dividendo social, puesto que es justamente la sociedad quien garantiza su propia existencia, es decir, de lo que se trata es de satisfacer una obligación social.

Otras definiciones de Patrocinio dicen que es:

Defender, proteger, amparar, favorecer...

Sufragar una empresa, con fines publicitarios, los gastos de un programa de radio o televisión, de una competición deportiva o de un concurso.

Acuerdo entre un patrocinador y un patrocinado, con beneficios recíprocos, para que cada uno consiga sus propios objetivos.

Instrumento publicitario que hace posible ligar directamente una marca o una empresa a un acontecimiento atrayente para un público dado.

Contribución prestada a una persona, manifestación, producto u organización, a cambio del beneficio directo plasmado en el derecho a incluir publicidad de marca que, de esta forma, se asocia a la obra o manifestación patrocinada y destacar así de cualquier otra publicidad.

Es mantener un evento, actividad, persona u organización suministrando dinero $\mathrm{u}$ otros recursos a cambio de algo, generalmente, publicidad.

Sostén aportado a una manifestación, a una persona, a un producto o a una organización co vistas a obtener un beneficio directo. 
Ayuda o aportación económica, en especie, en personal, equipamientos, tecnología, etc., de una persona jurídica o física (el patrocinador) a una actuación, entidad o persona (la patrocinada) de naturaleza cultural, científica, de solidaridad, deportiva, etc., ajena a la empresa, con el compromiso de que al patrocinador le suponga alguna contrapartida, no económica, generalmente en el campo de la imagen y la comunicación, asociada a la actuación o a la entidad patrocinada.

Técnica publicitaria más sofisticada que la elemental emisión de un anuncio o la colocación de una valla, gracias a la cual se relaciona la marca de una compañía o producto con un espacio radiofónico, televisivo, un acontecimiento deportivo o un evento cultural. (Octavio Rojas Orduña)

Inversión de una entidad para amparar o favorecer un evento, con cargo al presupuesto de comunicación de la misma, habitualmente encuadrado en un plan de marketing con objetivos de venta.

Crear, hacer posible o facilitar un espectáculo que, por el hecho de serlo, pasa a los medios de comunicación llevando asociado el nombre de un patrocinador y produciendo, en consecuencia, un volumen de comunicación comercial que viene a compensarle la inversión.

Y otras definiciones de Mecenazgo dicen que es:

Protección dispensada por una persona a un escritor o artista.

Es el plus de poder y de influencia social que buscan ciertos empresarios, ya que, con el dinero sólo, nunca lo podrían conseguir. (Amado Juan de Andrés)

Acción efectuada por una persona física o moral que actúa en el campo cultural en el sentido del interés general, sin que tal acción esté vinculada a su actividad normal o haya sido impuesta por ésta.

Sostén financiero o material, aportado sin contrapartida directa por parte del beneficiario, a una obra o a una persona para el ejercicio de actividades que presenten un carácter de interés general. 
Práctica organizada a través de la cual una organización comercial o empresa destina recursos propios para la financiación parcial o total de un acontecimiento en el campo de los contenidos simbólicos, y que al estar revestido de ciertas condiciones específicas, actúe como soporte de un mensaje corporativo, esperando como contraprestación de su aporte, una mediatizada contribución a la elevación de su notoriedad e imagen en el entorno.

Actividad de Relaciones Públicas que consiste en la financiación y apoyo de actos e iniciativas sociales y culturales. Si bien tiene como finalidad provocar una imagen favorable de la entidad en la sociedad, en general, no persigue, de forma directa, una predisposición positiva de un público objetivo determinado hacia los productos o iniciativas de la empresa o entidad mecenas.

Instrumento precioso de comunicación y un medio de promoción cultural, en una palabra, un instrumento de integración de la empresa en la sociedad.

Como puede apreciarse existen tantas analogías como diferencias entre ambos conceptos, entre ambas técnicas. Aunque suelen ir casi siempre de la mano, entre el patrocinio y el mecenazgo hay diferencias sustanciales. Es cierto que ambos son instrumentos de creación de imagen y que las organizaciones tanto públicas como privadas obtienen importantes dividendos en lo que a imagen se refiere. Otra similitud es que ambos son también instrumentos de responsabilidad social como ya hemos avanzado y veremos más adelante, puesto que se trata de acciones voluntarias asumidas como obligaciones impuestas por la norma de cultura, es decir, por la sociedad. Sin embargo, mientras que el patrocinio tiene una alta motivación comercial, el mecenazgo consiste en preservar, conservar y fomentar el arte, la cultura y el patrimonio cultural. Además, el patrocinio es más un instrumento publicitario en el que cada parte logra una serie de objetivos. Por su parte, el mecenazgo es fundamentalmente promoción de valores y adquisición de reconocimiento. Otra distinción es que el patrocinio es mucho más directo que el mecenazgo, que es más discreto. Mientras que en las acciones de patrocinio la 
iniciativa parte del patrocinado que le propone a una organización pública o privada participar en ella; en las actividades de mecenazgo es el inversor, es decir, el patrocinador o mecenas quien toma las riendas por lo general.

En definitiva, el patrocinio supone un incremento rápido de la notoriedad, ya sea de la entidad o de un producto determinado. Además, se logra una mayor presencia en los medios de comunicación y una mejora de la imagen corporativa y de marca. Mientras que a través del Mecenazgo, las empresas estrechan relaciones con sus distintos públicos, se integran mucho mejor en la comunidad y sociedad y obtienen ventajas fiscales.

A pesar de sus diferencias tanto el Patrocinio como el Mecenazgo son técnicas de Relaciones Públicas, ya que se enmarcan en esos denominados procesos de comunicación específicos que las organizaciones ponen en marcha constantemente con objeto de relacionarse son sus públicos para así lograr esa cohesión social que pretenden las Relaciones Públicas. El ejercicio de las Relaciones Públicas son un conjunto de actividades, es decir, procesos de comunicación, que las organizaciones llevan a cabo, primero, para darse a conocer; y segundo, para justificar su existencia, pues es la sociedad quien la garantiza. Es decir, lo que las empresas tanto públicas como privadas pretenden es lograr ser socialmente aceptadas y ello sólo es posible a través de la actividad publirrelacionista, donde se ubican, entre otras muchas actividades, el Patrocinio y el Mecenazgo.

\section{Patrocinio y Mecenazgo, instrumentos de Responsabilidad Social Corporativa}

Es sabido que el Mecenazgo es el primer precedente histórico de Responsabilidad Social que se conoce, como se ha citado en líneas más arriba. ¿Y qué se entiende por Responsabilidad Social? ¿Y por Responsabilidad Social Corporativa? En primer lugar, cabe destacar que pocos temas existen más controvertidos y polémicos en el 
ámbito de las Relaciones Públicas como es el de la Responsabilidad Social Corporativa.

Entendemos por responsabilidad social el conjunto de obligaciones inherentes a la libre asunción de un estado o condición, aún no reconocidas por el ordenamiento jurídico positivo o reconocidas sólo parcialmente, pero cuya fuerza vinculante y su previa tipificación proceden de la íntima convicción social de que su incumplimiento constituye una infracción de la norma de cultura.

La responsabilidad social se resuelve en un conjunto de obligaciones que se concretan en un dar, hacer o no hacer alguna cosa, de acuerdo con el clásico concepto ofrecido por el Derecho Romano, conectado a la asunción -por supuesto, libre y voluntaria- de un estado o condición socialmente trascendente y que por ello determina un comportamiento normado; segundo, ese conjunto de obligaciones constitutivo de un comportamiento que aún no ha sido refrendado por el ordenamiento jurídico positivo, por lo que no es exigible; tercero, ese conjunto de obligaciones tiene fuerza vinculante e incluso, sanción, dado que tiene a un sujeto como punto final de imputación y una norma como referencia; en el supuesto de infracción, la sociedad pone en marcha su complejo mecanismo sancionador, cuyo espectro de posibilidades es inmenso; cuarto, esa fuerza vinculante halla su fundamento legitimador en el grupo social y en la valoración que el mismo realiza de los intereses en presencia, de acuerdo con la "norma de cultura". El mundo empresarial, el "mundo de los negocios", en el decir angloamericano, sostenía y me atrevo a afirmar que sostiene en la actualidad, la responsabilidad social de la empresa, es decir, una responsabilidad que trasciende los angostos límites de la contenida en los textos legales que regulan la actividad industrial y mercantil y demás normas complementarias y que abarcan multitud de aspectos. Los economistas, al menos un sector suficientemente significativo de ellos, limitan esa responsabilidad al campo económico, afirmando que la responsabilidad de la empresa consiste, ni más ni menos, en la obtención del máximo beneficio, sin que se le pueda atribuir ninguna otra obligación, excepción hecha, claro está, de las de 
carácter fiscal, laboral, etc., a las que habría que añadir las que la presión social de la hora presente va cargando sobre sus "espaldas" (respeto del medio ambiente, lucha contra la contaminación, etc.). No obstante y aunque aparentemente ambas posturas divergen radicalmente, la realidad no es así, ya que sus fronteras distan mucho de ofrecer perfiles nítidos y claramente diferenciados, supuesto que sus fundamentos se hallan en ámbitos dispares, obedientes las más de las veces a concepciones políticas, sociales y religiosas inspiradoras de unas filosofías vitales de carácter emocional o intuitivo y desde luego, de muy difícil racionalización, siquiera se nos presenten o pretendan presentar rodeadas de un halo de "cientificismo". La razón de esa discordancia entre apariencia y realidad se halla en el hecho de que la idea e incluso el concepto de responsabilidad social se han ceñido exclusivamente o casi exclusivamente al "mundo de los negocios", esto es, al ámbito empresarial, más concretamente a la empresa, olvidando la existencia de otros campos cuya importancia no precisa ser subrayada. De ahí que nosotros pretendamos desvincularla de esas limitaciones y referirnos a los entes colectivos formales, expresión preferible a nuestro juicio a la generalmente utilizada, traductora de un pragmatismo muy difícil de conjugar con la visión generalista y universal del fenómeno. A fin de cuentas y no obstante su importancia o trascendencia, la empresa constituye tan sólo una mera anécdota en el acontecer humano, un simple modo de producción, organización y distribución de bienes y servicios, surgido históricamente. De ahí que propongamos hablar de entes colectivos formales, expresión susceptible de abarcar tanto las empresas, o entidades con ánimo de lucro, como todas aquellas organizaciones sin ánimo de lucro, pero que, no obstante, se ven obligadas a rendir socialmente cuentas de su actividad, ya que en cualquier caso manejan unos recursos humanos y materiales escasos. En cualquier caso y como inevitable concesión al uso de los tiempos, nos vemos obligados, como observará el lector, a referirnos constantemente a la empresa y no como sería nuestro gusto a los entes colectivos formales, expresión ésta más genérica y en consecuencia, más adecuada. Sin duda, se trata de un tema polémico, siquiera ya esté admitido por gran 
parte de la doctrina y no sólo naturalmente en nuestro campo, sino en el mucho más espinoso de la ciencia económica, en la que se ha abierto paso paulatinamente. En gran medida, resulta sorprendente que fuera el mundo del Derecho el primero que dio el gran paso y en una institución que, desde Roma, se había considerado perfectamente cristalizada: el derecho de propiedad. Para el romano, en efecto, la propiedad era el "ius utendi, fruendi et abutendi", es decir, el derecho de usar, disfrutar e incluso abusar de las cosas. En palabras infinitamente más elegantes y sin perder un ápice de rigor, nuestros grandes romanistas lo definían diciendo que era "el señorío jurídico sobre las cosas". Sin embargo, el derecho de propiedad, incluso en tiempos muy primitivos, estuvo sujeto a diversas limitaciones que, con el paso de los tiempos, fueron perfilándolo hasta convertirlo en una sombra de lo que antaño fue. La propiedad se concibe actualmente como un derecho social, por encima del cual se hallan los intereses de la sociedad, que priman siempre y en todo caso sobre él. Autores ha habido que niegan su existencia, considerándolo una enfiteusis. Sea como fuere, es lo cierto que la concepción del derecho de propiedad influyó sobremanera en la idea de la responsabilidad social que estudiamos. La empresa y haciendo abstracción de otros factores no desdeñables como, verbigracia, su consideración de ente artificial, cuya única justificación se halla en su consideración de servir del modo más idóneo para el fin para el que fue creada, no es ni puede ser nunca un ente absoluto ni en su constitución ni en su desenvolvimiento. La empresa está al servicio de la sociedad porque maneja recursos humanos y materiales naturalmente escasos, recursos que detrae del cuerpo social y que al dedicarlos a la producción de bienes o servicios determinados significa también que impide su aplicación a otros menesteres. Una analogía casi caricaturesca nos lo demuestra. La responsabilidad social corporativa no debe estimarse jamás como una opción voluntaria por parte de las corporaciones, sino como una obligación ineludible que, en el futuro, será jurídicamente exigible incluso ante los órganos jurisdiccionales. Cuando se habla de responsabilidad social corporativa, es como si se tratara de una manifestación actitudinal defensiva ante circunstancias intolerables: la defensa del 
entorno, el salario justo, la defensa de los intereses del usuario o consumidor, etc. Ello es correcto y no hay nada que añadir; sin embargo, nosotros entendemos que hay que dar un paso adelante y dejar de configurar la responsabilidad social desde puntos de vista reactivos, sino activos, lo cual se corresponde con la figura, todavía escasamente desarrollada, del ciudadano corporativo. En efecto, la empresa, la corporación, si se quiere, ha experimentado un grado de crecimiento tal que ha superado todas las previsiones; ya no es concebible la empresa que limita sus actividades a un mercado local, regional o incluso, nacional; sus acciones han desbordado fronteras y no sólo en el ámbito económico, sino también en el político; su poder económico ha desestabilizado gobiernos $y$ ha impuesto, en ocasiones, gobiernos dictatoriales, usurpando la voluntad y la capacidad de decisión de los pueblos. No hace falta recordar el caso de Chile, en el que la ITT “derribó" al legítimo gobierno de Allende, apoyando al general "golpista" Augusto Pinochet e imponiendo una dictadura presumiblemente acorde con sus intereses. Este formidable poder alcanzado por las mal llamadas empresas multinacionales -más adecuadamente deberían denominarse "transnacionales"-, siquiera tengan como nadie ignora una nacionalidad perfectamente identificada e identificable, es decir, sean plenamente "nacionales"-, no sólo ha afectado al mundo político y económico, sino también y es posiblemente lo más grave, a la sociedad, siquiera sean los aspectos antes mentados los más sobresalientes por más visibles.

\section{Conclusiones}

El Patrocinio y Mecenazgo forman parte de ese conjunto de obligaciones, pues se trata de requerimientos sociales, necesidades sociales que las organizaciones deben satisfacer, ya que de lo contrario se infringiría la norma de cultura, lo que no les reportaría dividendos en imagen. Es decir, la imagen pública es consecuencia directa del grado de asunción de responsabilidad social que expresa con su conducta una empresa o, mejor dicho, del grado de convicción social acerca de su cumplimiento, y 
ello coincide con el fundamento las propias Relaciones Públicas, es decir, la aceptación social está en función del comportamiento de esta naturaleza que no es otro, sino el correspondiente al comportamiento esperado. $\mathrm{O}$ dicho de otra forma, aquella organización que acomode su comportamiento a los requerimientos sociales, de acuerdo con la norma de cultura de la sociedad en la que se desenvuelva, disfrutará de una imagen buena, en el sentido de ser reconocida como valiosa.

\section{Bibliografía}

Cabra de Luna, Miguel Ángel (1998): “El tercer sector y las Fundaciones en España hacia el nuevo milenio. Enfoque económico, social y jurídico". Madrid.

Cuesta, Marta de la y Galindo, Angel (Coords.) (2005): “Inversiones socialmente responsables".

Cuesta, Marta de la y Rodríguez Duplá, Leonardo (coords.) (2004): “Responsabilidad Social Corporativa".

Cuesta, Marta de la, Valor Martínez, Mamen y Sanmartín Serrano, Sergio (2002): "Inversiones éticas en empresas socialmente responsables".

Fernández Daza, Eliseo (1995): “Estudio y análisis de los aspectos económicos, financieros y de control de las Fundaciones". Madrid.

Fundación Empresa y Sociedad (1997): “La estrategia social de la empresa. En enfoque de valor". Madrid.

Fundación Empresa y Sociedad (1999): “Casos de empresas que apoyan el empleo de personas desfavorecidas". Madrid. 
Fundación Empresa y Sociedad (2003): “De la transparencia a la confianza”.

G. Perdiguero, Tomás (2003): “La responsabilidad social de las empresas en un mundo global".

Gimeno Ullastres, Juan Antonio (1995): “Sector público y patrocinio”.

Juan de Andrés, Amado (1993): "Menenazgo y Patrocinio, las claves del Marketing". Madrid.

Martín Fernández, Javier (1996): "Régimen tributario del mecenazgo en España". Madrid.

Martínez, Juan Luis, Simón, Cristina y Agüero, Ana (2003): “La acción social de la empresa".

Mayor Zaragoza, Federico (1993): “El papel de las Fundaciones en el desarrollo de la sociedad civil". Santander.

Minguella Rubio, Francesca (1995): “Menenazgo empresarial: de pasiones y de intereses". Madrid.

Minguella, Francesca; Aguilá, Oriol; Rabanal, Marc (2000): “El patrocinio y mecenazgo de empresa en su contexto". Aedme. Madrid.

Ministerio de Cultura (1992): "El patrocinio empresarial de la cultura en España". Madrid. 
Montoso Chiner, María Jesús (1997): “Patrocinio Privado y público en el renacer del Gran Teatro del Liceo", Barcelona.

Pares i Maicas, Manuel (1991): "La Nueva Filantropía y la comunicación social". Barcelona.

Rabanal, Marc (2004): “El Patrocinio y Mecenazgo empresarial, en el marco de la Responsabilidad Social Corporativa"

Rodríguez Fernández; José Miguel: “El gobierno de la empresa. Un enfoque alternativo".

Rodríguez, Delfín (et al.) (1995): “Mecenazgo y conservación del patrimonio artístico: reflexiones sobre el caso español". Madrid.

Vicente Domingo, Elena (1998): “El contrato de Esponsorización” Madrid.

Vidal Portabales, José Ignacio: “El contrato de patrocinio publicitario en el Derecho español". Madrid. 